\title{
Passive Presence Detection Algorithm for Wi-Fi Sensing
}

\author{
Artyom LOMAYEV ${ }^{1}$, Claudio R. C. M. DA SILVA ${ }^{2}$, Alexander MALTSEV ${ }^{1,3}$, \\ Carlos CORDEIRO ${ }^{2}$, Ali S. SADRI ${ }^{4}$ \\ ${ }^{1}$ Intel Corp., Nizhny Novgorod, 603024, Russia \\ ${ }^{2}$ Intel Corp., Hillsboro, OR, 97124, USA \\ ${ }^{3}$ University of Nizhny Novgorod, Nizhny Novgorod, 603950, Russia \\ ${ }^{4}$ Intel Corp., San Diego, CA, 92121, USA \\ artyom.lomayev@intel.com
}

Submitted February 27, 2020 / Accepted July 28, 2020

\begin{abstract}
In this paper, we derive a signal processing algorithm that enables a Wi-Fi station to passively detect the presence of a potential user in its vicinity. It is assumed that the potential user either doesn't carry a Wi-Fi device or, if it does, that its device does not participate in the detection procedure. Passive presence detection is performed by the station by means of tracking over time channel estimates obtained with packets transmitted by one or more stations in the Wi-Fi network, and determining when the user presence impacts the received signals. The proposed algorithm performs binary hypothesis testing and decides if a potential user is in the vicinity of the Wi-Fi station. It uses an estimate of the dynamic channel component power as the test statistic and compares it to a predefined threshold. As formulated in the paper, to increase the detection reliability, the power of the dynamic channel component is maximized by using an optimization procedure. Experimental results obtained with off-the-shelf $\mathrm{Wi}$ $F i$ devices and with the proposed algorithm are presented which demonstrate the validity of the analytical formulation, as well as the feasibility of performing passive presence detection using a Wi-Fi network. In controlled residential and enterprise settings, the proposed algorithm provided a detection rate of $99.7 \%$ for a false alarm rate of less than $1 \%$.
\end{abstract}

\section{Keywords}

Passive presence detection, motion detection, Wi-Fi sensing, binary hypothesis testing, channel state information

\section{Introduction}

Wi-Fi sensing is a term given to the use of traditional Wi-Fi technology to perform radar-like applications such as detecting motion in a room or detecting when a potential user approaches a Wi-Fi-enabled device. A key advantage of using Wi-Fi to enable sensing applications over other technologies, including infrared sensors and video cameras, is the possibility of re-using systems that are already widely deployed in both home and enterprise environments for data communications to provide new applications and services.

Research on Wi-Fi sensing has been ongoing at least since the early 2000s. Applications considered so far include motion and presence detection, people counting, activity classification, vital sign monitoring, and gesture recognition, among many others. The literature in the area is vast and growing, and we refer the interested reader to [1-5] for surveys of the field. In November 2019, IEEE 802.11 created a Study Group (SG) focused on Wi-Fi sensing, with the expectation that it will result in a standard-development activity. As discussed in [6-12], standard support is critical to enable Wi-Fi devices and networks to better serve sensing related applications and spur further innovation.

In applications such as motion and presence detection, Wi-Fi sensing is performed by tracking channel estimates obtained with multiple Wi-Fi packets, which could have been sent by one or more devices over time and detecting changes that may indicate an event of interest. This is because as a person or object moves, it impacts the wireless signal propagation in the environment (that is, propagation paths are created and cancelled generating time-varying multipath fading). In addition, if more than one wireless link exists (for example, when a Wi-Fi station receives beacon frames from multiple access points), it is expected that the links will exhibit different, but possibly correlated, time-dependent variation patterns as a result of motion. Therefore, the overall performance of $\mathrm{Wi}-\mathrm{Fi}$ sensing is expected to improve when signals transmitted by more than one Wi-Fi station (access points, for example) are used. The algorithm derived here exploits this hypothesis.

The purpose of the algorithm derived in this paper is to enable a Wi-Fi station to passively detect the presence of a potential user in its proximity ( $\sim 1-2 \mathrm{~m}$ radius). Presence detection is an important application of $\mathrm{Wi}-\mathrm{Fi}$ sensing, and 
of interest in areas such as system wake-up and environment monitoring. By passive, we mean that the "subject" (the person that enters a room in a motion detection application, for example) doesn't carry a Wi-Fi device or, if it does, that its device does not participate in the sensing procedure.

The paper is organized as follows. In Sec. 2, we describe the system model to be considered. The proposed algorithm is then derived in Sec. 3 by maximization of power for the dynamic channel component, which is used as a test statistic in the binary hypothesis testing. In Sec. 4, experimental results obtained with off-the-shelf Wi-Fi devices and with the derived algorithm are presented. The proposed algorithm performance is compared to the algorithms recently published in the literature, [13-15]. Conclusions are drawn in Sec. 5.

\section{System Model}

We denote the device that uses Wi-Fi sensing to detect the presence of a potential user in its proximity by sensing station, and the one or more Wi-Fi stations that transmit signals used by the sensing station as transmit stations. To perform passive presence detection, it is assumed that transmit stations send packets with a certain regularity that are received and processed by the sensing station. Channel estimates are obtained in the frequencydomain by using the Long Training Field (LTF) within the Wi-Fi Orthogonal Frequency Division Multiplexing (OFDM) modulated packet preamble, [16].

Let $\mathbf{H}_{l}$ denote the frequency domain $N_{\mathrm{F}} \times N_{\mathrm{ST}}$ channel matrix obtained by the sensing station when using packets transmitted by the $l^{\text {th }}$ transmit station. Specifically,

$$
\mathbf{H}_{l}=\left[\begin{array}{cccc}
H_{l, 0,0} & H_{l, 0,1} & \ldots & H_{l, 0,\left(N_{\mathrm{ST}}-1\right)} \\
H_{l, 1,0} & H_{l, 1,1} & \ldots & H_{l, 1,\left(N_{\mathrm{ST}}-1\right)} \\
\vdots & \vdots & \ddots & \vdots \\
H_{l,\left(N_{\mathrm{F}}-1\right), 0} & H_{l,\left(N_{\mathrm{F}}-1\right), 1} & \ldots & H_{l,\left(N_{\mathrm{F}}-1\right),\left(N_{\mathrm{ST}}-1\right)}
\end{array}\right]
$$

where $H_{l, i, j}$ is the complex channel estimate obtained for the $j^{\text {th }}$ subcarrier of the $i^{\text {th }}$ packet transmitted by the $l^{\text {th }}$ transmit station, and $j=0,1, \ldots, N_{\mathrm{ST}}-1, i=0,1, \ldots, N_{\mathrm{F}}-1$, and $l=$ $0,1, \ldots, N_{\mathrm{L}}-1$. The parameters $N_{\mathrm{ST}}, N_{\mathrm{F}}$, and $N_{\mathrm{L}}$ denote the total number of OFDM subcarriers in each received packet, the number of successively received packets, and the number of links (e.g., transmit stations) being tracked, respectively.

The channel matrix $\mathbf{H}_{l}$ can be decomposed into a static component $\mathbf{H}_{\mathrm{s}, l}$ and a dynamic component $\mathbf{H}_{\mathrm{d}, l}$,

$$
\mathbf{H}_{l}=\mathbf{H}_{\mathrm{s}, l}+\mathbf{H}_{\mathrm{d}, l} .
$$

The static component $\mathbf{H}_{\mathrm{s}, l}$ corresponds to the average of the channel computed with respect to the column (time) dimension of the matrix $\mathbf{H}_{l}$ defined in (1) and represents the part that does not evolve in time at the duration of $N_{\mathrm{F}}$ consecutive packets. We introduce an estimate of the $H_{\mathrm{s}, l, i, j}$ component as a sample mean value in the form:

$$
H_{\mathrm{s}, l, i, j}=\frac{1}{N_{\mathrm{F}}} \sum_{k=0}^{N_{\mathrm{F}}-1} H_{l, k, j} .
$$

The dynamic component $\mathbf{H}_{\mathrm{d}, l}$ consists of the correlated channel variation in time caused by the motion of the "subject" (human or object) with reference to the static component. In a perfectly stationary environment, $\mathbf{H}_{\mathrm{d}, l}=0$. In contrast, in a perfectly dynamic environment $\mathbf{H}_{\mathrm{s}, l}=0$. These two scenarios describe limit cases, and in practice both components are not equal to zero.

A detector associates the non-zero dynamic channel component with motion in the vicinity of the detector, while the static channel component does not carry information related to motion detection and as shown next, has no impact. If one uses a sample covariance of $\mathbf{H}_{l}$, denoted by $\mathbf{C}_{\mathbf{H}_{l}}$, it can remove the estimate of stationary component from the original channel matrix $\mathbf{H}_{l}$ and estimate the dynamic component only. By definition,

$$
\begin{aligned}
& \mathbf{C}_{\mathbf{H}_{l}}=\operatorname{cov}\left(\mathbf{H}_{l}\right)=\frac{1}{N_{\mathrm{F}}-1}\left(\mathbf{H}_{l}-\mathbf{H}_{\mathrm{s}, l}\right)^{\mathrm{H}}\left(\mathbf{H}_{l}-\mathbf{H}_{\mathrm{s}, l}\right) \\
& =\operatorname{cov}\left(\mathbf{H}_{\mathrm{d}, l}\right)=\frac{1}{N_{\mathrm{F}}-1} \mathbf{H}_{\mathrm{d}, l}^{\mathrm{H}} \mathbf{H}_{\mathrm{d}, l}
\end{aligned}
$$

where $\mathbf{C}_{\mathbf{H}_{l}}$ is the $N_{\mathrm{ST}} \times N_{\mathrm{ST}}$ sample covariance matrix of the $N_{\mathrm{F}} \times N_{\mathrm{ST}}$ channel matrix $\mathbf{H}_{l}$.

The received signal for the $l^{\text {th }}$ transmit link $\mathbf{S}_{\mathrm{R}, l}$ can be written in the form:

$$
\mathbf{S}_{\mathrm{R}, l}=\mathbf{H}_{l} \mathbf{S}_{\mathrm{T}}+\mathbf{N}_{l}
$$

where $\mathbf{S}_{\mathrm{T}}$ is the $N_{\mathrm{ST}} \times N_{\mathrm{ST}}$ diagonal pilot matrix and $\mathbf{N}_{l}$ is the $N_{\mathrm{F}} \times N_{\mathrm{ST}}$ complex Additive White Gaussian Noise (AWGN) matrix with zero mean and variance $\sigma_{N}{ }^{2}$. The pilot matrix $\mathbf{S}_{\mathrm{T}}$ consists of the LTF modulated symbols defined in [16].

The output of the channel estimator operating under noisy conditions for the $l^{\text {th }}$ transmit link is defined as:

$$
\mathbf{Y}_{l}=\mathbf{H}_{l} \mathbf{S}_{\mathrm{T}} \mathbf{S}_{\mathrm{T}}^{\mathrm{H}}+\mathbf{N}_{l} \mathbf{S}_{\mathrm{T}}^{\mathrm{H}}=\mathbf{H}_{l}+\mathbf{Z}_{l}, \mathbf{S}_{\mathrm{T}} \mathbf{S}_{\mathrm{T}}^{\mathrm{H}}=\mathbf{I}, \mathbf{Z}_{l}=\mathbf{N}_{l} \mathbf{S}_{\mathrm{T}}^{\mathrm{H}}(6)
$$

where $\mathbf{Z}_{l}$ is the $N_{\mathrm{F}} \times N_{\mathrm{ST}}$ noise matrix with zero mean and variance $\sigma_{\mathrm{Z}}{ }^{2}$. The received matrix $\mathbf{S}_{\mathrm{R}, l}$ is multiplied by the inverse transmit pilot matrix, which is equal to the Hermitian conjugate of the original matrix $\mathbf{S}_{\mathrm{T}}$ (see [16]). Note that the impact of the carrier frequency offset and the timing sampling offset should be compensated before $\mathbf{Y}_{l}$ can be used by the presence detection algorithm.

Using (2)-(6) and the sample covariance matrix definition, the sample covariance matrix of $\mathbf{Y}_{l}$, denoted by $\mathbf{C}_{\mathbf{Y}}$, is written as

$$
\mathbf{C}_{\mathbf{Y}_{l}}=\frac{1}{N_{\mathrm{F}}-1}\left(\mathbf{H}_{\mathrm{d}, l}^{\mathrm{H}} \mathbf{H}_{\mathrm{d}, l}+\mathbf{Z}_{l}^{\mathrm{H}} \mathbf{H}_{\mathrm{d}, l}+\mathbf{H}_{\mathrm{d}, l}^{\mathrm{H}} \mathbf{Z}_{l}+\mathbf{Z}_{l}^{\mathrm{H}} \mathbf{Z}_{l}\right) .
$$


The obtained sample covariance matrix $\mathbf{C}_{\mathbf{Y} l}$ given by (7) is used to compute the scalar test statistic $Y$ of the detection algorithm. First, a two-dimensional frequency domain filtering is applied by multiplication on vector $\mathbf{V}_{l}$ of size $N_{\mathrm{ST}} \times 1$ on the right and its Hermitian conjugate on the left side of matrix $\mathbf{C}_{\mathbf{Y} /}$. Second, a spatial filtering is applied by combining different transmit links together by weighting on $W_{l}$ coefficients of $N_{\mathrm{L}} \times 1$ vector $\mathbf{W}$ :

$$
Y=\sum_{l=0}^{N_{\mathrm{L}}-1} W_{l} \mathbf{V}_{l}^{\mathrm{H}} \mathbf{C}_{\mathbf{Y}_{l}} \mathbf{V}_{l} .
$$

The derived scalar test statistic $Y$ represents an estimate of the aggregated dynamic channel power and quantifies the correlated channel variation in time of transmit links that are possibly also correlated in the space domain. This test statistic was used in the binary hypothesis testing in the algorithm for presence detection described in Sec. 3. The proposed algorithm applies optimization in the multidimensional space with respect to the vectors $\left(\mathbf{V}_{0}, \mathbf{V}_{1}, \ldots\right.$, $\left.\mathbf{V}_{N_{\mathrm{L}-1}}, \mathbf{W}\right)$ to enhance detection performance.

\section{Algorithm for Passive Presence Detection}

The proposed passive presence detection algorithm uses a binary hypothesis testing (see [17]), by comparing the test statistic $Y$, introduced in (8), to a threshold $\gamma$. If the test statistic is smaller than the threshold, the algorithm determines that there is no presence ( $H_{0}$, null hypothesis). Conversely, if the test statistic is greater than or equal to the threshold, the algorithm indicates the presence of a potential user in the vicinity of sensing station $\left(H_{1}\right.$, alternative or signal hypothesis).

The scalar test statistic $Y$ can be decomposed into two components:

$$
Y=X+Z
$$

where $X$ corresponds to the signal component (that is, the dynamic component of the channel) and $Z$ is the noise component. Substituting (7) into (8) and using (9), we have

$$
\begin{aligned}
& X=\frac{1}{N_{\mathrm{F}}-1} \sum_{i=0}^{N_{\mathrm{L}}-1} W_{l} \mathbf{V}_{l}^{\mathrm{H}} \mathbf{H}_{\mathrm{d}, l}^{\mathrm{H}} \mathbf{H}_{\mathrm{d}, l} \mathbf{V}_{l}, \\
& Z=\frac{1}{N_{\mathrm{F}}-1} \sum_{i=0}^{N_{\mathrm{L}}-1} W_{l} \mathbf{V}_{l}^{\mathrm{H}}\left(\mathbf{Z}_{l}^{\mathrm{H}} \mathbf{H}_{\mathrm{d}, l}+\mathbf{H}_{\mathrm{d}, l}^{\mathrm{H}} \mathbf{Z}_{l}+\mathbf{Z}_{l}^{\mathrm{H}} \mathbf{Z}_{l}\right) \mathbf{V}_{l} .
\end{aligned}
$$

Under the $H_{0}$ null hypothesis and with the assumption that the channel is perfectly static $\left(\mathbf{H}_{\mathrm{d}, l}=0\right), X$ is equal to 0 and

$$
Z=\frac{1}{N_{\mathrm{F}}-1} \sum_{i=0}^{N_{\mathrm{L}}-1} W_{l} \mathbf{V}_{l}^{\mathrm{H}} \mathbf{Z}_{l}^{\mathrm{H}} \mathbf{Z}_{l} \mathbf{V}_{l}
$$

Under the $H_{1}$ signal hypothesis, $X$ is greater than zero and $Z$ cannot be further simplified from the general form given in (10) and includes two additional noise cross-term components. As a result, the mean value and standard deviation for $H_{1}$, denoted as $E_{1}$ and $\sigma_{1}$, respectively, are greater than the $E_{0}$ and $\sigma_{0}$ counterparts defined for $H_{0}$.

The proposed algorithm performs multi-dimensional maximization of $Y$ variable with respect to vectors $\left(\mathbf{V}_{0}, \mathbf{V}_{1}\right.$, $\left.\ldots, \mathbf{V}_{N_{\mathrm{L}-1}}, \mathbf{W}\right)$. In case of the null hypothesis $H_{0}$, the input to the algorithm is the noise matrix $\mathbf{Z}_{l}$ only. Due to the random nature of $\mathbf{Z}_{l}$, and statistical independence of its components in the time domain and over different transmit links, the maximization does not lead to significant power enhancement. In contrast, for hypothesis $H_{1}$, the dynamic channel component $\mathbf{H}_{\mathrm{d}, l}$ consists of the correlated channel variation in time and possible correlation of different transmit links in space. As a result, the maximization leads to a significant power enhancement and a better differentiation (or deflection) between both hypotheses and increasing the detector reliability.

The test statistic $Y$ is always greater than or equal to zero due to symmetry of $\mathbf{Y}_{l}$ and its meaning as a power estimate. Thus, the maximization of $Y$ is equivalent to the maximization of $J$, which is a square function of it:

$$
\begin{aligned}
& J\left(\mathbf{V}_{0}, \mathbf{V}_{1}, \ldots, \mathbf{V}_{N_{\mathrm{L}}-1}, \mathbf{W}\right)=\left(\sum_{l=0}^{N_{\mathrm{L}}-1} W_{l} \mathbf{V}_{l}^{\mathrm{H}} \mathbf{C}_{\mathbf{Y}_{l}} \mathbf{V}_{l}\right)^{2} \rightarrow \max _{\mathbf{V}_{l}, \mathbf{W}}, \\
& \left\|\mathbf{V}_{l}\right\|^{2}=1,\|\mathbf{W}\|^{2}=1, l=0,1, \ldots, N_{\mathrm{L}}-1 .
\end{aligned}
$$

The maximization of the quadratic form in (12) is performed in the multi-dimensional vector space with respect to vectors $\left(\mathbf{V}_{0}, \mathbf{V}_{1}, \ldots, \mathbf{V}_{N_{\mathrm{L}^{-1}}}, \mathbf{W}\right)$ and is subject to constraint that all vectors have a unit power. The maximization of the quadratic form $J$ is simpler than the maximization of $Y$ because it can be solved by applying standard optimization algorithms, [18].

To solve (12), we use the Lagrange dual function approach, by augmenting the objective function $J\left(\mathbf{V}_{0}, \mathbf{V}_{1}, \ldots\right.$, $\left.\mathbf{V}_{N_{\mathrm{L}-1}}, \mathbf{W}\right)$ with the $N_{\mathrm{L}}+1$ constraint functions weighted by the Lagrange scalar multipliers $\left(\lambda_{0}, \lambda_{1}, \ldots, \lambda_{N_{\mathrm{L}-1}}, \lambda_{W}\right)$, [18]:

$$
\begin{aligned}
& L\left(\mathbf{V}_{0}, \mathbf{V}_{1}, \ldots, \mathbf{V}_{N_{\mathrm{L}}-1}, \mathbf{W}, \lambda_{0}, \lambda_{1}, \ldots, \lambda_{N_{\mathrm{L}}-1}, \lambda_{W}\right) \\
& =\left(\sum_{l=0}^{N_{\mathrm{L}}-1} W_{l} \mathbf{V}_{l}^{\mathrm{H}} \mathbf{C}_{\mathbf{Y}_{l}} \mathbf{V}_{l}\right)^{2}-\sum_{l=0}^{N_{\mathrm{L}}-1} \lambda_{l}\left(\mathbf{V}_{l}^{\mathrm{H}} \mathbf{V}_{l}-1\right)-\lambda_{W}\left(\mathbf{W}^{\mathrm{H}} \mathbf{W}-1\right) .
\end{aligned}
$$

Taking the derivative of (13) with respect to each variable, including vectors $\left(\mathbf{V}_{0}, \mathbf{V}_{1}, \ldots, \mathbf{V}_{N_{\mathrm{L}-1}}, \mathbf{W}\right)$ and scalar multipliers $\left(\lambda_{0}, \lambda_{1}, \ldots, \lambda_{N_{\mathrm{L}-1}}, \lambda_{W}\right)$, and forcing them to zero, results in the following system of equations: 




where the $N_{\mathrm{L}} \times 1$ vector $\mathbf{B}$ is introduced to simplify the notation. Its $l^{\text {th }}$ element is defined as

$$
B_{l}=\mathbf{V}_{l}^{\mathrm{H}} \mathbf{C}_{\mathbf{Y}_{l}} \mathbf{V}_{l} \text {. }
$$

Solving the first two equations in (14), we obtain

$$
\mathbf{W}_{\text {opt }}=\mathbf{B} / \sqrt{\mathbf{B}^{\mathrm{H}} \mathbf{B}}, \lambda_{W, \mathrm{opt}}=\mathbf{B}^{\mathrm{H}} \mathbf{B} .
$$

Substituting (16) into the quadratic form in (12) gives us

$$
\left(\sum_{l=0}^{N_{\mathrm{L}}-1} W_{l, \mathrm{opt}} \mathbf{V}_{l}^{\mathrm{H}} \mathbf{C}_{\mathbf{Y}_{l}} \mathbf{V}_{l}\right)^{2}=\sum_{l=0}^{N_{\mathrm{L}}-1}\left(\mathbf{V}_{l}^{\mathrm{H}} \mathbf{C}_{\mathbf{Y}_{l}} \mathbf{V}_{l}\right)^{2}=\lambda_{W, \text { opt }} .
$$

From (17), we have that the optimization over $\mathbf{W}$ results in the decoupling of the links and makes further optimization over the $\mathbf{V}_{l}$ vectors independent. Therefore, substituting (16) into (14), we obtain

$$
\left(\mathbf{V}_{l}^{\mathrm{H}} \mathbf{C}_{\mathbf{Y}_{l}} \mathbf{V}_{l}\right) \mathbf{C}_{\mathbf{Y}_{l}} \mathbf{V}_{l}=\lambda_{l} \mathbf{V}_{l}, \mathbf{V}_{l}^{\mathrm{H}} \mathbf{V}_{l}=1
$$

The solution for $\mathbf{V}_{l}$ is one of the eigenvectors of $\mathbf{C}_{\mathbf{Y} l}$, and the Lagrange multiplier $\lambda_{l}$ is equal to the square of the corresponding eigenvalue, [19]:

$$
\mathbf{V}_{l, \mathrm{opt}}=\mathbf{Q}_{l}^{(m)}, \lambda_{l, \mathrm{opt}}=\left(\Lambda_{l}^{(m)}\right)^{2}, \Lambda_{l}^{(m)}=\left(\mathbf{Q}_{l}^{(m)}\right)^{\mathrm{H}} \mathbf{C}_{\mathbf{Y}_{l}} \mathbf{Q}_{l}^{(m)},
$$

for $m=0,1, \ldots, N_{\text {rank }, l}-1$, where $N_{\text {rank,l }}$ is the rank of $\mathbf{C}_{\mathbf{Y}_{l}}$. In (18), $\mathbf{Q}_{l}^{(m)}$ and $\Lambda_{l}^{(m)}$ are the $m^{\text {th }}$ eigenvector of $\mathbf{C}_{\mathbf{Y}_{l}}$ and its corresponding eigenvalue, respectively. The matrix eigenvalue decomposition can be found by applying a standard algorithm such as power iterations, [19].

Substituting (17) and (19) into (8), we obtain that the optimal test statistic is given by

$$
Y_{\mathrm{opt}}^{(m)}=\sqrt{\sum_{l=0}^{N_{\mathrm{L}}-1}\left(\Lambda_{l}^{(m)}\right)^{2}} .
$$

In words, the optimal test statistic has multiple solutions equal to the square root of the sum of the $m^{\text {th }}$ squared eigenvalues found for each sample covariance matrix $\mathbf{C}_{\mathbf{Y}_{l}}$. In the case when the system relies on measurements ob- tained with a single link $\left(N_{\mathrm{L}}=1\right)$, the optimal test statistic reduces to be an eigenvalue of the particular sample covariance matrix $\mathbf{C}_{\mathbf{Y} l}$. This particular case of the detector can be found in many papers, typically as a building block of more complex algorithms, for instance, please see [14], [15].

Note, that the particular choice of the solution in (20) will affect the $E_{0}$ and $\sigma_{0}$ estimates and therefore affects the detection performance. To combine the multiple solutions into a single variable, a normalized test statistic is introduced:

$$
u=\frac{1}{N_{\text {rank }}} \sum_{m=0}^{N_{\text {rank }}{ }^{-1}} \frac{Y_{\mathrm{opt}}^{(m)}-E_{0}^{(m)}}{\sigma_{0}^{(m)}}
$$

where $N_{\text {rank }}=\min _{l}\left(\operatorname{rank}\left(\mathbf{C}_{\mathbf{Y} l}\right)\right)$ and $Y_{\text {opt }}{ }^{(m)}$ is given by (20). The $E_{0}{ }^{(m)}$ and $\sigma_{0}{ }^{(m)}$ are the mean value and standard deviation of $Y_{\mathrm{opt}}{ }^{(m)}$ found under $H_{0}$ null hypothesis. Both $E_{0}{ }^{(m)}$ and $\sigma_{0}{ }^{(m)}$ can be estimated during calibration period interval as will be further discussed in Sec. 4 .

The normalized test statistic $u$ defined in (21) represents a weighted sum of the test statistics corresponding to the different solutions shifted by the $E_{0}{ }^{(m)}$ mean value and weighted to the inverse of the $\sigma_{0}{ }^{(m)}$ value. The larger the values of $E_{0}{ }^{(m)}$ and $\sigma_{0}{ }^{(m)}$, the smaller is the component's contribution to the resulting statistic. This test statistic was used in the conducted experimental measurements to detect presence event in the residential home environment described in Sec. 4.

\section{Experimental Results}

To demonstrate the feasibility of passive presence detection using Wi-Fi networks and the validity of the proposed algorithm, we conducted a large number of experiments using off-the-shelf Wi-Fi devices from multiple vendors in typical residential and enterprise environments. In this paper, we present results obtained with measurements made on the second floor of a single-family home in Portland, OR. The scenario considered is illustrated in Fig. 1. In Fig. 1, the red squares (labeled TX STA 1, TX STA 2, and TX STA 3) represent the transmit stations (as defined in Sec. 2), and the green square (SENS STA) represents the sensing station.

Each transmit station was configured to send $20 \mathrm{MHz}$ packets with a $100 \mathrm{~ms}$ interval. The sensing station receives packets from all transmit stations and estimates the channel with each received packet as a part of the standard data detection procedure. To calculate the sample covariance matrix $\mathbf{C}_{\mathbf{Y} l}$ for each link, we took $N_{\mathrm{F}}$ and $N_{\mathrm{ST}}$ to be equal to 20 packets and 52 (data and pilot) subcarriers, respectively (see [16]). The parameter $N_{\mathrm{L}}$ in (20) was set equal to 3 and $N_{\text {rank }}$ in (21) to 16 .

Changes in the environment were created by the movement of a single person with the trajectory depicted in Fig. 1. As shown in the figure, the subject was first motionless in the office for the first $30 \mathrm{~s}$ of the experiment to per- 


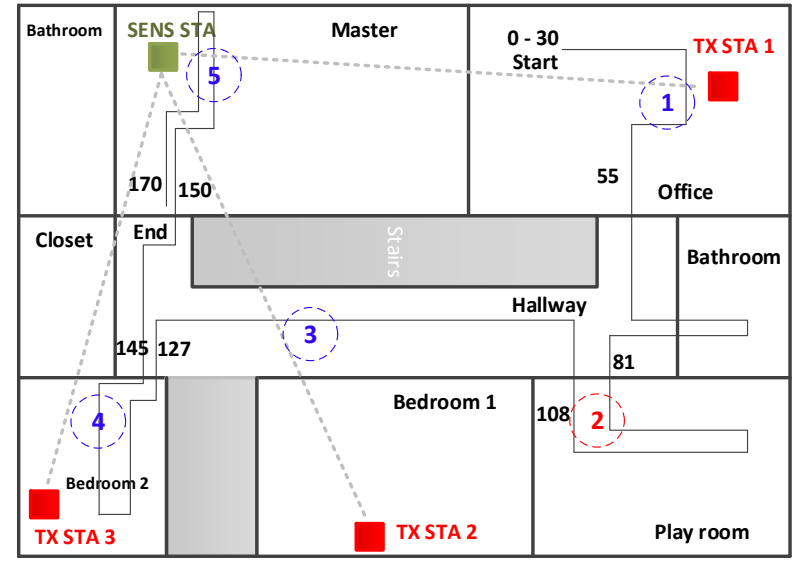

Fig. 1. Floor plan and motion trajectory in experiment \#1.

form algorithm calibration by means of estimating the parameters $E_{0}^{(m)}$ and $\sigma_{0}{ }^{(m)}$ used in (21); then walked around the same room for $25 \mathrm{~s}$, and subsequently left the office. After that, the subject went in and out of a bathroom, and entered the playroom at $t=81 \mathrm{~s}$ and stayed there for $27 \mathrm{~s}$. After leaving the playroom, the subject went down the hallway towards bedroom 2 , went in $(t=127 \mathrm{~s})$ and out $(t=145 \mathrm{~s})$ of bedroom 2 , and finally entered the master bedroom, where the sensing station is located, at $t=150 \mathrm{~s}$. Upon entering the master, the subject walked towards the sensing station, and stayed in its proximity (within 2 meters) until the end of the experiment.

In Fig. 1, marker 1 indicates motion in the proximity of TX STA 1, marker 2 corresponds to a "blind spot" where motion was not detected, markers 3 and 4 indicate motion in the proximity of TX STA 2 and TX STA 3, respectively, and marker 5 indicates proximity to the sensing station SENS STA.

The output of the proposed presence detection algorithm, which represents a normalized test statistic $u$ introduced in (21) and plotted as a function of time for the scenario and experiment described in Fig. 1 is given in Fig. 2. The outputs corresponding to the five events shown in Fig. 1 (markers 1-5) are also noted in Fig. 2. A number of important observations can be made when comparing both figures.

First, the test statistic $u$ is not as large when the subject is close to one of the transmit stations (markers 1,3 , and 4) compared to when the subject is close to the sensing station (marker 5). It can be concluded that the receiverbased scheme is more sensitive to motion than the transmitter-based scheme and the intensity of the reaction decreases with increasing of the distance between the moving person and the receiver. Additionally, for the configuration considered in the experiment, only one of the links used for Wi-Fi sensing is impacted at a time when the movement is close to a transmit station, while all links are impacted (spatially correlated) when movement is close to the sensing station.

The presence of a potential user in the proximity of the sensing station could thus be obtained by comparing

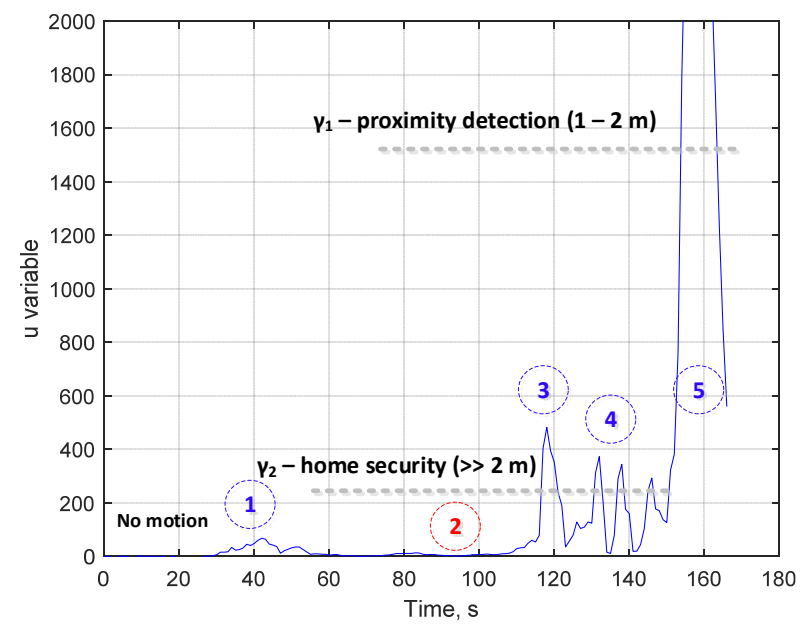

Fig. 2. Normalized test statistic plotted as a function of time for motion trajectory in experiment $\# 1$.

test statistic $u$ with a threshold $\gamma$ that is large. In Fig. 2 this corresponds to the $\gamma=\gamma_{1}$ example, where the threshold value can be set between 600 and 2000. Using the experimental measurements, we determined that a threshold in this range leads to a detection range of approximately 1-2 $\mathrm{m}$ from the sensing station. Setting the threshold to a higher value may reduce the impact of the potential "background moving" problem for proximity detection, which may occur when there is more than one person present in the coverage area of the Wi-Fi sensing application.

Second, while the proposed algorithm was derived for proximity detection, it is seen in Fig. 2 that it could also be used for other applications, such as home security by means of motion detection. To this end, the threshold would have to be set to a lower value than the one used for proximity detection so that motion indicated by just one of the links triggers detection. In Fig. 2 this corresponds to the $\gamma=\gamma_{2}$ example, where the threshold can be set to the value below 400 , which corresponds to a detection range greater than $2 \mathrm{~m}$. In that case, the system would detect the motion events denoted by markers 3 and 4 . In general, by setting the threshold to appropriate values, we can adjust the target distance from the sensing station where we would like to detect an event of presence and support different applications.

Lastly, as expected, the performance of the algorithm depends on the number of transmit stations and on their relative position with respect to each other and to the sensing station. The "blind spot" indicated by marker 2 , which would be problematic in a home security application, for example, could be addressed by placing a transmit station at the hallway or by changing the location of the existing transmit stations, among other options.

A second experiment is shown in Fig. 3 for a case when the potential user approaches the sensing station multiple times. The trajectory and markers shown in the figure should be interpreted in the same way as those shown in Fig. 1. The output of the proposed algorithm (normalized test statistic $u$ ) for this new experiment is given in Fig. 4. 


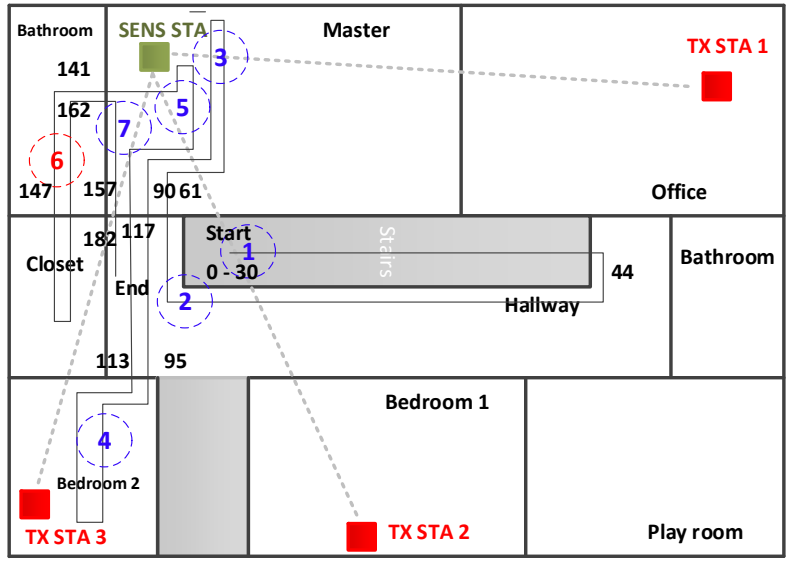

Fig. 3. Floor plan and motion trajectory in experiment \#2.

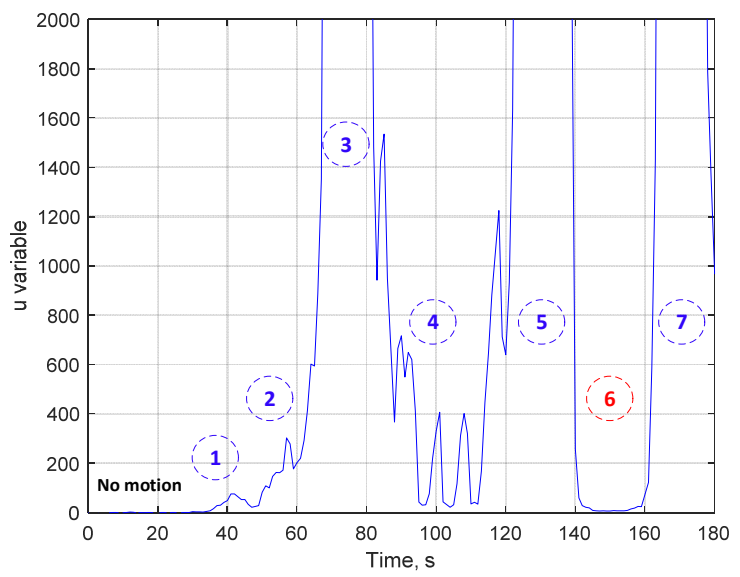

Fig. 4. Normalized test statistic plotted as a function of time for motion trajectory in experiment $\# 2$.

Similar to what was observed in Fig. 2, larger values of test statistic $u$ can be reliably used to detect the presence of a potential user close to the sensing station.

In both experimental setups shown in Fig. 1 and Fig. 3, we used interval of $30 \mathrm{~s}$ at the beginning of each motion depicted as "no motion" in Fig. 2 and Fig. 4, where environment was completely static to perform parameters calibration. In realistic Wi-Fi operation, such intervals will not be available and the case that the environment is static cannot be guaranteed with certain reliability. Therefore, in practical systems, such calibration can be performed using a number of LTF training fields following each other inside the single packet. The channel estimates will not change much over consecutive LTFs inside the packet due to very short relative time delay and therefore may be considered as static. Using these LTFs algorithm can perform $E_{0}{ }^{(m)}$ and $\sigma_{0}{ }^{(m)}$ parameters estimation and support self-calibration procedure. However, currently, standard does not define such a training field (see [16]), and additional work is required to define a new frame format in the future standard.

The extensive measurements conducted in the typical but controlled residential and enterprise environments have shown that the proposed algorithm provides a detection rate of $99.7 \%$ for a false alarm rate of less than $1 \%$. Figure 5 shows a summary of the results, where the $\mathrm{Y}$ axis



Fig. 5. Proposed algorithm performance and its comparison to the known methods.

corresponds to the detection rate in $\%$ and the $\mathrm{X}$ axis refers to the type of algorithm.

As a performance reference, we considered the algorithms recently published in the literature [13-15]. In reference [13], the authors suggest an algorithm for human motion detection based on the channel frequency response estimation using successively received packets in time. The channel measurements were conducted in a conference room environment with a single transmit link. The authors introduced a short-term averaged variance ratio (SVR) and a long-term averaged variance ratio (LVR) test statistic in the form:

$$
R_{\mathrm{SVR}}=\frac{1}{n} \sum_{i=1}^{n}\left|\frac{\delta_{\Delta T}^{i}}{\delta_{\Delta T-1}^{i}}\right|, R_{\mathrm{LVR}}=\frac{1}{n} \sum_{i=1}^{n}\left|\frac{\delta_{\Delta T}^{i}}{\delta_{\Delta L T}^{i}}\right|, \delta_{\Delta T}^{i}=\frac{\sigma_{\Delta T}^{i}}{\mu_{\Delta T}^{i}}
$$

where $\sigma_{\Delta T}^{i}$ is the standard deviation and $\mu_{\Delta T}^{i}$ is the mean value of the channel phase estimated for the $i^{\text {th }}$ subcarrier over short and long observation time intervals $\Delta T$ and $\Delta L T$, respectively, and $n$ is the total number of subcarriers equal to 30 in the experiment. The introduced test statistics are compared to the threshold to detect human motion. It was shown that the algorithm can achieve $95 \%$ detection rate.

In reference [14], the authors proposed an algorithm for human motion detection based on a Support Vector Machine (SVM) learning algorithm. Similar to [13], the algorithm estimates channel frequency responses over successively received packets in time. The channel measurements were conducted in different types of environments, including a meeting room, a classroom, a hallway, and a laboratory with a single transmit link. The eigenvalues of the covariance matrix for the amplitudes and phases are used as the test statistics:

$$
\alpha=\operatorname{eigen}(\mathbf{R}(|\mathbf{H}|)), \rho=\operatorname{eigen}(\mathbf{R}(\arg (\mathbf{H})))
$$

where $\mathbf{R}(|\mathbf{H}|)$ and $\mathbf{R}(\arg (\mathbf{H}))$ are the covariance matrices of the channel amplitudes and phases, accordingly. The first two eigenvalues for the amplitudes $\left(\alpha_{1}, \alpha_{2}\right)$ and phases $\left(\rho_{1}, \rho_{2}\right)$ are combined into the feature vector and used as an input to the SVM algorithm. A supervised learning is 
applied to train an SVM algorithm to classify the static and dynamic channel state. It was shown that the algorithm can achieve $99 \%$ detection rate.

In reference [15], the authors suggested an algorithm for human motion detection based on a machine learning approach. In addition to the SVM algorithm used in [14], a Random Forest (RF) and a K-Nearest Neighbor (KNN) algorithm were also considered. The channel frequency response measurements were conducted in different types of environment, including a meeting room, a hallway, and a laboratory with a single transmit link. The eigenvalues of the covariance matrix for the amplitudes and phases defined in (23) are used as the test statistics. The authors consider a feature vector, which consists of the amplitude eigenvalues only, a mixture of both amplitude and phase eigenvalues, and the phase eigenvalues only. The detection rate for the different algorithms and feature vectors was analyzed. It was shown that the best detection rate of $99.8 \%$ can be achieved with the RF algorithm and the phase eigenvalues feature vector.

It can thus be concluded that the proposed algorithm achieves a similar detection rate as a machine learning approach for a user proximity detection application. In the proposed algorithm, a complex matrix processing is performed without separation between the amplitudes and phases of the channel realization. An improved detection reliability is achieved with the use of channel realizations from the multiple transmit links. In contrast to the previous works, the introduced normalized test statistic in (21), selects the dominant eigenvalues in the decomposition by means of weights adaptation in the resulting sum. The algorithm does not require a training period when deployed in a new environment, as required by most machine learning-based approaches.

\section{Conclusions}

In this paper, we derived a signal processing algorithm that enables a Wi-Fi device to passively detect the presence of a potential user in its vicinity. In the proposed algorithm, detection is performed by the device by means of tracking channel estimates obtained with packets transmitted by one or more stations in the Wi-Fi network over time and determining when the user presence impacts the received signals. Experimental results demonstrated both the validity of the presented analytical formulation and the feasibility of performing passive presence detection using a Wi-Fi network.

\section{References}

[1] MA, Y., ZHOU, G., WANG, S. WiFi sensing with channel state information: A survey. ACM Computing Surveys, 2019, vol. 52, no. 3, p. 1-36. DOI: $10.1145 / 3310194$
[2] MA, J., WANG, H., ZHANG, D., et al. A survey on Wi-Fi based contactless activity recognition. In Proceedings of the 2016 International IEEE Conferences on Ubiquitous Intelligence \& Computing, Advanced and Trusted Computing, Scalable Computing and Communications, Cloud and Big Data Computing, Internet of People, and Smart World Congress. Toulouse (France), 2016, p. 1086-1091. DOI: 10.1109/UIC-ATC-ScalCom-CBDCom-IoPSmartWorld.2016.0170

[3] JIANG, H., CAI, C., MA, X., et al. Smart home based on WiFi sensing: A survey. IEEE Access, 2018, vol. 6, p. 13317-13325. DOI: 10.1109/ACCESS.2018.2812887

[4] WANG, Z., JIANG, K., HOU, Y., et al. A survey on CSI-based human behavior recognition in through-the-wall scenario. IEEE Access, 2019, vol. 7, p. 78772-78793. DOI: 10.1109/ACCESS.2019.2922244

[5] LIU, J., LIU, H., CHEN, Y., et al. Wireless sensing for human activity: A survey. IEEE Communication Surveys and Tutorials, early access article, 2019, p. 1-17. DOI: 10.1109/COMST.2019.2934489

[6] HAN, T. X., DU, R., LIU, C., et al. Wi-Fi sensing. IEEE Document 802.11-19/1164r0, Jul. 2019. [Online] Available at: https://mentor.ieee.org/802.11/documents?is_dcn=1164\&is_group =0wng

[7] DA SILVA, C., CORDEIRO, C., SADEGHI, B., et al. Wi-Fi sensing: Usages, requirements, technical feasibility and standards gaps. IEEE Document 802.11-19/1293r0, Jul. 2019. [Online] Available https://mentor.ieee.org/802.11/documents?is_den=1293\&is_group $=0 \mathrm{wng}$

[8] DA SIlva, C., CORDEIRO, C., SADEGHI, B., et al. Wi-Fi sensing: Cooperation and standard support. IEEE Document 802.11-19/1416r0, Sept. 2019. [Online] Available at: https://mentor.ieee.org/802.11/documents?is_dcn=1416\&is_group =0wng

[9] HAN, T. X., DU, R., ZHOU, B., et al. Wi-Fi sensing-Follow-up. IEEE Document 802.11-19/1500r0, Sept. 2019. [Online] Available at: https://mentor.ieee.org/802.11/documents?is_dcn=1500\&is_group $=0 \mathrm{wng}$

[10] EITAN, A., KASHER, A., TRAININ, S. Wi-Fi sensing in $60 \mathrm{GHz}$ band. IEEE Document 802.11-19/1551r1, Sept. 2019. [Online] Available https://mentor.ieee.org/802.11/documents?is_dcn=1551\&is_group $=0 \mathrm{wng}$

[11] AU, O., WANG, B., LIU, K. J. R., et al. 802.11 sensing: Applications, feasibility, standardization. IEEE Document 802.1119/1626r1, Sept. 2019. [Online] Available at: https://mentor.ieee.org/802.11/documents?is_dcn=1626\&is_group =0wng

[12] UNTERHUBER, P., SCHMIDHAMMER, M., SAND, S. Wi-Fi sensing application: Multipath enhanced device free localization. IEEE Document 802.11- 19/1580r0, Sept. 2019. [Online] Available https://mentor.ieee.org/802.11/documents?is_dcn=1580\&is_group =0wng

[13] GONG, L., YANG, W., MAN, D., et al. WiFi-based real-time calibration-free passive human motion detection. Sensors, 2015, vol. 15 , no. 12 , p. $32213-32229$. DOI: $10.3390 / \mathrm{s} 151229896$

[14] QIAN, K., WU, C., YANG, Z., et al. Enabling contactless detection of moving humans with dynamic speeds using CSI. ACM Transactions on Embedded Computing Systems, Jan. 2018, vol. 17 , no. 2 , p. 1-18. DOI: $10.1145 / 3157677$

[15] DING, E., LI, X., ZHAO, T., et al. A robust passive intrusion detection system with commodity WiFi devices. Journal of Sensors, 2018, p. 1-12. DOI: 10.1155/2018/8243905 
[16] IEEE SA. Wireless LAN Medium Access Control (MAC) and Physical Layer (PHY) Specifications, IEEE Std. 802.11-2016, Dec. 2016.

[17] KAY, S. M. Fundamentals of Statistical Signal Processing Detection Theory, vol. 2. Englewood Cliffs (NJ): Prentice-Hall, 1998. ISBN: 0-13-345711-7

[18] BOYD, S., VANDENBERGHE, L. Convex Optimization. Cambridge University Press, 2004. ISBN: 978-0-521-83378-3

[19] STRANG, G. Introduction to Linear Algebra. Wellesley (MA): Cambridge Press, 2009. ISBN: 978-0-9802327-1-4

\section{About the Authors ...}

Artyom LOMAYEV was born in Nizhny Novgorod, Russia, in 1983. He received the M.S. degree in Electrical Engineering from the Nizhny Novgorod State University, Nizhny Novgorod, Russia, in 2005. From 2005 to 2008, he was a Research Assistant with the Bionics and Statistical Department at Nizhny Novgorod State University. Since 2006, he has been a Research Scientist with the Next Generation and Standards group, Intel Corporation. He is the author of 20 articles, more than 80 inventions, and 150+ contributions to the IEEE $802.11 \mathrm{ad} / \mathrm{ay}$ standards. His interests include digital signal processing, information theory, estimation and detection theory, channel coding theory, and PHY/MAC layer design for wireless systems.

Claudio R. C. M. DA SILVA is a systems engineer with the Wireless Connectivity Standards group of Intel Corporation and has several years of experience in wireless communication system design, prototyping, and implementation. He has been an active contributor to various IEEE 802.11 activities, including the development of IEEE 802.11ay (Next Generation $60 \mathrm{GHz}$ ) and the creation of a WLAN sensing effort. After receiving a $\mathrm{Ph} . \mathrm{D}$. degree in Electrical Engineering from the University of California, San Diego in 2005, he first worked as an assistant professor at Virginia Tech and later became a member of Samsung Mobile Solutions Lab, when he was engaged in cellular modem implementation and applied research. He was an editor for IEEE Transactions on Communications and has served on the technical program committee of numerous IEEE conferences in the communications area.

Alexander MALTSEV received the Candidate of Science degree and the Doctor of Science degree, both in Radiophysics, from the University of Nizhny Novgorod
(UNN), Russia, in 1975 and 1990, respectively. From 1994 till present he is holding the position of Head of the Statistical Radiophysics Department in the UNN. In April 2001 Prof. Maltsev joined Intel Corporation and created Advance Development (AD) team from his former $\mathrm{PhD}$ students in Intel Russia. From April 2006 till present he is an Intel Principal Engineer managing AD team. During that time, he contributed to development of Wi-Fi (IEEE802.11n, 11ad), WiMAX (IEEE802.16e, 16m), WiGig and LTE standards. He participated in FP6 MEMBRANE project (2006-2009) and EU-Japan FP7 MiWEBA project (2013-2016), headed the Russian Evaluation Group in IMT-Advanced (4G) evaluation process in ITU (2010-2011). Prof. Maltsev is author of numerous papers in refereed journals and conferences proceedings and holds about 150 US patents. His research interests include optimal and adaptive statistical signal processing, adaptive antenna arrays, MIMO-OFDM communication systems including $5 \mathrm{G}$.

Carlos CORDEIRO is the CTO of wireless connectivity at Intel Corporation, where he is responsible for next generation wireless connectivity technology strategy, standards, ecosystem engagements, and regulatory. In the $\mathrm{Wi}-\mathrm{Fi}$ Alliance, he is a member of the Board of Directors and serves as its Technical Advisor. Due to his contributions to wireless communications, Dr. Cordeiro received several awards including the prestigious Intel Inventor of the Year Award in 2016, the 2017 IEEE Standards Medallion, the IEEE Outstanding Engineer Award in 2011, and the IEEE New Face of Engineering Award in 2007. Dr. Cordeiro is the co-author of two textbooks on wireless published in 2006 and 2011, has published over 110 papers in the wireless area alone, and holds over 250 patents. Dr. Cordeiro is an IEEE Fellow.

Ali S. SADRI is the General Manager of mmWave Standards and Advanced Technology at Intel Corporation. Ali has more than 30 years of scientific and engineering background starting at IBM, Duke University and at Intel since 2002. Dr. Sadri has demonstrated successful track record of building breakthrough technologies such as WiGig $60 \mathrm{GHz}$ standards, mmWave mesh Backhaul and disruptive mmWave Distributed Relay. Dr. Sadri has over 150 patents all in wired line and wireless communications system. Ali serves as an industry advisor to Tokyo Institute of Technology and a technical advisor to the WISE program for Super Smart Society. 